\title{
Noncommutativity in Space and Primordial Magnetic Field
}

\author{
Anupam Mazumdar and Mohammad M. Sheikh-Jabbari \\ The Abdus Salam International Center for Theoretical Physics, Strada Costiera 11, Trieste, Italy
} (Received 16 January 2001; published 19 June 2001)

In this paper we show that noncommutativity in spatial coordinates can generate magnetic field in the early Universe on a horizon scale. The strength of such a magnetic field depends on the number density of massive charged particles present at a given moment. This allows us to trace back the temperature dependence of the noncommutativity scale from the bounds on primordial magnetic field coming from nucleosynthesis.

DOI: $10.1103 /$ PhysRevLett.87.011301

Although the idea of having a noncommutative spacetime is an old one [1], until a renewed motivation from string theory [2], it has not been studied seriously. The operators corresponding to coordinates of a noncommutative space (Moyal plane), $\hat{x}^{\mu}$, satisfy

$$
\left[\hat{x}^{\mu}, \hat{x}^{\nu}\right]=i \theta^{\mu \nu}
$$

where $\theta^{\mu \nu}$ is a given constant which has a dimension of [length] [2]. For our purposes it is more convenient to introduce the noncommutativity scale, $\Lambda_{\mathrm{NC}}$, given by

$$
\theta^{\mu \nu}=\frac{c^{\mu \nu}}{\Lambda_{\mathrm{NC}}^{2}},
$$

where $c^{\mu \nu}$ is an antisymmetric tensor whose components are $\mathcal{O}(1)$. In order to obtain the noncommutative version of a given field theory, generally one can use the following prescription. Take the classical action for the field theory and replace the product of the fields by a $\star$-product, such as

$$
\begin{aligned}
(f \star g)(x) & =\left.\exp \left(\frac{i}{2} \theta_{\mu \nu} \partial_{x_{\mu}} \partial_{y_{\nu}}\right) f(x) g(y)\right|_{x=y} \\
& =f(x) g(x)+\frac{i}{2} \theta_{\mu \nu} \partial_{\mu} f \partial_{\nu} g+\mathcal{O}\left(\theta^{2}\right) .
\end{aligned}
$$

We notice the $\star$-product in Eq. (3) is a noncommutative one, $f \star g \neq g \star f$. For some useful relations on the $\star$-product calculus, see Ref. [3]. It has been noticed that there are problems with the unitarity and causality in the noncommutative space-time if $\theta_{0 i} \neq 0$ [4]. Therefore, in this paper we restrict ourselves to the noncommutative space, i.e., $\theta_{0 i}=0$.

Studying various physical consequences of such noncommutativity in spatial coordinates is of great interest. In this regard both quantum mechanical systems and field theory results can be used to constrain the lower bound on the noncommutativity scale. For the noncommutative quantum mechanics, the hydrogen atom in a noncommutative space has been discussed in Ref. [5], and its spectrum has also been calculated up to first order in $\theta$. There it is shown that noncommutativity lifts the degeneracy of some states in the spectrum and in particular it changes the Lamb-shift. For a general quantum mechanical system
PACS numbers: 98.80.Cq, 02.40.Gh, 11.25.Sq

with electromagnetic interactions the Hamiltonian receives a correction due to noncommutativity [5]. Such a correction at tree level up to first order in $\theta$ is given by assigning a momentum dependent electric dipole moment; $\vec{d}_{e}$ [6]

$$
d_{e}^{i}=\frac{e}{2 \hbar} \theta^{i j} p_{j} .
$$

The noncommutative field theories have their own attractions. The very basic question of the renormalizability of these field theories has been discussed in many papers; for some relevant references, see Ref. [7]. It has been shown that noncommutative version of a real $\phi^{4}$ theory is renormalizable up to two loops [3,8,9]. The noncommutative QED (NCQED) has also been discussed in Refs. [6,10], and shown to be renormalizable at one loop level. It was noticed that unlike the usual QED, NCQED is an asymptotic free theory. However, $\beta$ function does not depend on $\theta$ [10] (in general the $\theta \rightarrow 0$ limit is not a smooth one [3]).

By treating the noncommutative space as a natural extension to the usual space, many authors have studied the phenomenological consequences. By considering various $2 \rightarrow 2$ scattering processes in NCQED, and comparing the cross sections with that of the usual standard model, some lower bounds on the noncommutativity scale have been obtained in Refs. [11,12]. Here, we would like to mention the crucial point that in the noncommutative models the Lorentz invariance is explicitly broken. As a result of that, all the amplitudes are frame dependent. According to the results obtained from studying the cross sections, the lower bound on noncommutativity has been confirmed to be around 1000-2000 GeV [11]. Besides the scattering processes, the hydrogen atom spectrum and the Lamb shift can also be used to put some bounds on $\theta$, which leads to $\Lambda_{\mathrm{NC}} \gtrsim 10^{4} \mathrm{GeV}$ [5]. One can still obtain better bounds on $\Lambda_{\mathrm{NC}}$ up to $10^{5} \mathrm{GeV}$ from the neutron electric dipole moment [13].

As an interesting property of noncommutative space, it has been noticed in Ref. [6] that a magnetic dipole moment of a charged massive particle (e.g., electron) at one loop level receives some quantum corrections, which unlike the usual commutative case is spin independent, and it is directly proportional to $\theta$. Now, this can have an interesting 
consequence at a macroscopic level and a direct implication in the early Universe, such as generating magnetic fields at a cosmological scale. As we shall see the primordial magnetic fields constrain $\theta$, and this may improve the lower bound on a noncommutativity scale. As mentioned earlier, in the noncommutative space the Lorentz symmetry is violated, and in fact this magnetic field is also frame dependent. Our setup is good for a very slowly moving frame, i.e., what we actually have on the Earth, in which the isotropic cosmos assumption is a valid one.

As discussed earlier in Ref. [6], in NCQED the magnetic dipole moment of noncommutative Dirac particle at one loop is given by

$$
\langle\vec{\mu}\rangle=\frac{e q}{2 m} g \vec{S}+\frac{e \alpha \gamma_{e}}{6 \pi} q^{3} m \vec{\theta},
$$

where $\gamma_{\mathrm{e}} \sim 0.57$ denotes the Euler number, $m$ is the mass of a particle which carries charges $e q, \vec{S}$ is the spin of the particle, vector $\vec{\theta}$ is defined as $\theta_{i}=\epsilon_{i j k} \theta^{i j}$, and the gyromagnetic factor is denoted by $g$, which at one loop is given by

$$
g=2+\frac{\alpha q^{2}}{\pi}, \quad \alpha=\frac{e^{2}}{4 \pi}=\frac{1}{137} .
$$

The first term in Eq. (5) is the usual (commutative) expression, while the second term is due to noncommutativity and it is zero for a massless particle. Another important fact about the second term in Eq. (5) is unlike the first term, it is invariant under $\mathrm{C}$ (charge conjugation) and $\mathrm{CP}$. This can be understood by noticing that under $\mathrm{C}$ (and $\mathrm{CP}$ ) [14]

$$
\theta \stackrel{\mathrm{C}}{\rightarrow}-\theta, \quad \theta \stackrel{\mathrm{CP}}{\rightarrow}-\theta .
$$

Now, in order to obtain a large scale magnetic field originating from the matter content in a given volume, we must sum over all the possible states of the particles as

$$
\vec{B}=\sum_{i, \vec{S}}\left\langle\vec{\mu}_{i, \vec{S}}\right\rangle n_{i},
$$

where $i$ runs over all the "relevant set" of particles, where $n_{i}$ is the corresponding number density. The above expression for the magnetic field is quite interesting and as we have mentioned earlier, such a magnetic field spread in a large scale can be originated very easily in the early Universe. Especially, there exist large scale magnetic fields in galaxies and galaxy clusters with a large coherence length and a typical strength of $10^{-6} \mathrm{G}$, at a redshift of $z=0.395$ [15]. It is usually assumed that there must be a galactic dynamo mechanism which can amplify the preexisting primordial magnetic field. A recent study in this direction suggests that a weak magnetic field of strength $B_{g} \sim 10^{-30} \mathrm{G}$ during the galaxy formation can be sufficient to amplify the strength up to the present limit, provided there is a nonvanishing cosmological constant [16]. By now, there are many mechanisms which claim to understand the existence of the primordial magnetic field, acting as a seed. For a nice review, we refer the readers to Ref. [17].
However, the magnetic field due to the presence of noncommutativity has a marked difference. First of all the generation of this magnetic field is continuous and accumulative in nature. All it demands is the presence of charged massive particles present in any corner of the Universe. For such relativistic species the number density behaves as $n \approx T^{3}$, where $T$ is the temperature of the Universe. Thus we see that the strength of such a magnetic field depends on the temperature of the Universe. At this stage one might wonder what could be the strength of such a field at electroweak scale and at nucleosynthesis scale. It is a well-known fact that a strong magnetic field can alter thermodynamical distribution of the charged particles by modifying the phase space of volumes of the particles and the antiparticles, which can also affect the weak interaction processes. It was first noticed in Ref. [18] that a strong magnetic field can significantly enhance the $\beta$ decay rate of the neutrons. On this basis the authors have argued that relic abundance for ${ }^{4} \mathrm{He}$ will have a strong suppression compared to the standard case [19], because below the freezing temperature for the neutrons and protons, there is a finite time before they can coalesce to form a composite nuclei. In that finite time if the neutron abundance decreases due to its decay, it is natural that not only ${ }^{4} \mathrm{He}$ abundance is affected but also the abundances of heavier elements. Based on these facts the authors in Ref. [20] have derived constraints on the primordial magnetic field at the beginning of nucleosynthesis, corresponding to a temperature $T \sim 10 \mathrm{MeV} . \quad B(T=10 \mathrm{MeV})=10^{-8} \mathrm{GeV}^{2}$ for $Y_{p}=0.236$, where $Y_{p}$ denotes ${ }^{4} \mathrm{He}$ abundance. Thus it is important to check the validity of the present laboratory constraints on the scale of noncommutativity at a macroscopic level.

The "relevant set" of particles during nucleosynthesis could be $u, d$ quarks and electrons. Summation over spin of the particles is essentially zero, however, the spin independent part gives a nonvanishing magnetic field, which is given by

$$
\vec{B}=2 \frac{e \alpha \gamma_{\mathrm{e}}}{6 \pi} \times \vec{\theta} \times \sum_{i}\left(q_{i}^{3} m_{i}\right) n_{i} .
$$

Considering the net contribution of nucleons (proton and neutron) by their constituents $u, d$ quarks, and taking the masses of these quarks to be $\sim 5 \mathrm{MeV}$, we can estimate the net magnetic field, which yields

$$
B \approx\left(10^{-7}-10^{-8}\right) \times \theta \times n_{B} \mathrm{GeV}^{2},
$$

where for rough estimation we have replaced $n_{i}$ by the baryon number density $n_{B}$ at the time of nucleosynthesis. Knowing that a successful nucleosynthesis requires $n_{B} / n_{\gamma} \approx 10^{-10}$, where $n_{\gamma}=\left[2 \zeta(3) / \pi^{2}\right] T^{3}$ is the photon number density. Taking all these into account along with the scale of noncommutativity $\Lambda_{\mathrm{NC}} \sim 10^{3} \mathrm{GeV}$, we get a magnetic field strength during nucleosynthesis $B(T=10 \mathrm{MeV}) \sim 10^{-31} \mathrm{GeV}^{2}$, which is much weaker to cause any kind of threat to nucleosynthesis. Thus, the 
global presence of noncommutativity is hardly felt during nucleosynthesis. In fact, similar calculation can be repeated at later stages of the evolution also.

One can repeat a similar analysis during the galaxy formation at temperature $T_{\mathrm{gf}} \approx 10^{-3} \mathrm{eV}$, and, we get the number density of baryons in the horizon, $n_{B} \approx$ $10^{-41} \mathrm{GeV}^{3}$, which leads to net magnetic field with a strength $B_{\mathrm{gf}} \approx 10^{-34} \mathrm{G}$. This is again weaker compared to $10^{-20}-10^{-30} \mathrm{G}$, required for the amplification by the dynamo process to the present magnetic field in the galaxies. This again illustrates that perhaps the presence of noncommutativity can hardly influence the global structure of the Universe. It can be easily verified that the present contribution to the magnetic field due to noncommutativity is again much smaller than $10^{-9} \mathrm{G}$, a lower bound from COBE observation which constraints the anisotropic stresses due to the presence of magnetic field [21]. The reason behind such a weak effect is due to the fact that $\theta \propto 1 / \Lambda_{\mathrm{NC}}^{2}$, any enhancement in the noncommutativity scale makes any observable global affect weaker in magnitude. For instance, for a given number density of charged massive particles the magnetic field is inversely proportional to $\Lambda_{\mathrm{NC}}^{2}$, and its contribution to energy momentum tensor is suppressed by $\Lambda_{\mathrm{NC}}^{4}$. However, things might change if we go beyond electroweak scale. This we discuss in the rest of this paper.

So far we have limited our discussion below electroweak scale, during electroweak scale there will be a little enhancement in the magnetic field due to higher temperature and mass of the top quark which will dominate the particle spectrum. For $\Lambda_{\mathrm{NC}}=10^{3} \mathrm{GeV}$, the strength of the magnetic field comes around $B_{\text {ew }} \sim 10^{-17} \mathrm{GeV}^{2}$. If magnetic flux conservation holds good so that the energy contribution due to the magnetic field acts as a source of radiation, then the lines of magnetic fields are frozen along with the expansion of the Universe and follow $B \propto T^{2}$ [22]. Therefore the magnetic field produced during the electroweak scale will have a strength $\sim 10^{-25} \mathrm{GeV}^{2}$ during nucleosynthesis. This number is at least six orders of magnitude better than the magnetic field produced due to noncommutativity during nucleosynthesis. This leads to an obvious suspicion that at higher temperatures perhaps we might be able to get some appreciable affects due to noncommutativity. However, to proceed with this we need to speculate an exact strength of the magnetic field.

At temperatures more than the electroweak scale (before the electroweak phase transition) all the fermions are massless, so they do not contribute to large scale magnetic fields. In the usual Higgs scenario the Higgs field is the only massive field before the electroweak phase transition. It may seem that, being neutral, Higgs is not entering into the game. However, one should note that the fluctuations of the upper component in the Higgs doublet will lead to a charged massive particle. These particles after the electroweak phase transition are absorbed in the zero helicity part of the massive gauge bosons, $W^{ \pm}$. So, we have ful- filled the required assumption of having massive charged particles. However, we note that Eq. (5) is for fermionic field. For the scalar fields, such as Higgs, we believe that we still maintain the structural form of Eq. (5), but with a different numeric factor. However, Eq. (5) will still serve our purpose for an order of magnitude calculation. So, for a rough estimation we consider the magnetic field is given by

$$
B \approx\left(10^{-3}-10^{-4}\right) \times \frac{1}{\Lambda_{\mathrm{NC}}^{2}} \times m_{\mathrm{H}} n_{\mathrm{H}},
$$

where $m_{\mathrm{H}}, n_{\mathrm{H}}$ are the Higgs mass and number density, respectively. It is worth noting that since the running of the coupling constant $(\alpha)$ with temperature is logarithmic, this will not change our analysis appreciably. In order to get the temperature dependent $m_{\mathrm{H}}$, we use the usual Higgs model (e.g., [23])

$$
V(\Phi)=-\frac{\mu}{2}\left[\left(\frac{T}{T_{\mathrm{ew}}}\right)^{2}-1\right] \Phi^{2}+\frac{\lambda}{4} \Phi^{4},
$$

where $\Phi$ is the Higgs doublet, $T_{\text {ew }}$ is the electroweak phase transition temperature, and the usual electroweak scale and data (like Higgs mass) is defined by a proper choice of the constants $\mu$ and $\lambda$. From Eq. (10), one can read the temperature dependent Higgs mass as a coefficient of the first term. For $T \gg T_{\mathrm{ew}}, m_{\mathrm{H}} \propto T$, and $n_{\mathrm{H}} \propto T^{3}$. Since, the magnetic field $B \propto T^{2}$, one can easily read off the temperature dependence of the $\Lambda_{\mathrm{NC}}$. In a more general discussion $m_{\mathrm{H}} n_{\mathrm{H}}$ in Eq. (9) can be replaced by the energy density of the massive charged particles, $\rho$. For a relativistic scalar particles $\rho \propto T^{4}$, which gives a simple expression for $\Lambda_{\mathrm{NC}}$ beyond electroweak scale

$$
\Lambda_{\mathrm{NC}} \gtrsim \epsilon T,
$$

where $\epsilon$ being a constant which in our Higgs model is of the order of $\epsilon \approx \mathcal{O}(10-100)$. The upper value of $\epsilon$ is fixed by demanding that the strength of the magnetic field is constrained via nucleosynthesis. However, the reader might have noticed that below the electroweak scale the temperature dependence of $\Lambda_{\mathrm{NC}}$ is $\propto T^{1 / 2}$, because the essential charged particles are baryons whose number density scales as $a^{-3}$, where $a$ is the cosmological scale factor. The temperature dependence of noncommutativity has also been conjectured in Ref. [24], and some cosmological consequences have been discussed. However, in their case they just assume this temperature dependence to be steplike.

Finally, on a speculative note we mention that it is possible to have GUT baryogenesis at a very high temperature in an inflationary model. A temperature which can be at least 2-3 orders of magnitude larger than the usual reheat temperature of the Universe [25]. Usually, the reheat temperature is bounded by gravitino over production during reheating and it is usually set to be less than $10^{9} \mathrm{GeV}$. In that case it is possible to produce a large magnetic field due to noncommutativity. If such models are taken seriously, 
then it is also possible to constrain $\Lambda_{\mathrm{NC}}$ from the upper bound on the strength of the magnetic field during nucleosynthesis. However, due to many cosmological reasons a low reheat temperature is favorable, and that is why we do not delve into the details of constraining $\Lambda_{\mathrm{NC}}$ from the very early Universe.

We summarize our paper by mentioning that the presence of noncommutativity at a scale $\Lambda_{\mathrm{NC}} \approx 10^{3} \mathrm{GeV}$ does not cause any serious problem at the cosmological scale. Considering the noncommutativity as a seed for the primordial magnetic field, we have studied the temperature dependence of $\Lambda_{\mathrm{NC}}$. We have shown that this temperature dependence goes as $T^{1 / 2}$ for $T \lesssim T_{\text {ew }}$ and for $T \gg T_{\text {ew }}$, $\Lambda_{\mathrm{NC}} \propto T$, where $T_{\mathrm{ew}}$ is the electroweak phase transition temperature. Although the magnetic field we have found for $\Lambda_{\mathrm{NC}} \approx 10^{3}$ is less than the required seed for the usual dynamo effect, it can align the particles spin so that they can result in a net magnetic field providing the seed [26].

However, the present constraints on temperature of the Universe beyond nucleosynthesis is so weakly constrained that we cannot specifically define the noncommutativity scale $\Lambda_{\mathrm{NC}}$ without invoking a particular model of high or low reheat temperatures. If we choose inflationary models with a high reheat temperature, then it is quite possible that noncommutativity will play an interesting role during that period.

This work is partially supported by the EC Contract No. ERBFMRX-CT 96-0090, and A.M. acknowledges the support of The Early Universe Network HPRN-CT2000-00152.

[1] H. Snyder, Phys. Rev. 71, 38 (1947).

[2] N. Seiberg and E. Witten, J. High Energy Phys. 9909, 032 (1999).

[3] A. Micu and M. M. Sheikh-Jabbari, J. High Energy Phys. 0101, 025 (2001).

[4] J. Gomis and T. Mehen, Nucl. Phys. B 591, 265 (2000); M. Chaichian, A. Demichev, P. Prešnajder, and A. Tureanu, Report No. hep-th/0007156.
[5] M. Chaichian, M. M. Sheikh-Jabbari, and A. Tureanu, Phys. Rev. Lett. 86, 2716 (2001).

[6] I. F. Riad and M. M. Sheikh-Jabbari, J. High Energy Phys. 0008, 045 (2000).

[7] C. P. Martin and D. Sanchez-Ruiz, Nucl. Phys. B598, 348 (2001).

[8] I. Ya. Aref'eva, D. M. Belov, and A. S. Koshelev, Phys. Lett. B 476, 431 (2000).

[9] S. Minwalla, M. Van Raamsdonk, and N. Seiberg, J. High Energy Phys. 0002, 020 (2000).

[10] M. Hayakawa, Report No. hep-th/9912167.

[11] J. L. Hewett, F. J. Petriello, and T. G. Rizzo, Report No. hep-ph/0010354.

[12] P. Mathews, Phys. Rev. D 63, 075007 (2001).

[13] G. Senjanovic and M. M. Sheikh-Jabbari (private communication).

[14] M. M. Sheikh-Jabbari, Phys. Rev. Lett. 84, 5265 (2000).

[15] P. P. Kronberg and J. J. Perry, Astrophys. J. 263, 518 (1982); P. P. Kronberg, J. J. Perry, and E. L. H. Zukowski, ibid. 387, 528 (1992).

[16] A. C. Davis, M. Lilley, and O. Törnkvist, Phys. Rev. D 60, 021301 (1999).

[17] For a review, see D. Grasso and H. R. Rubinstein, Report No. astro-ph/0009061.

[18] J. Matese and R.F. O'Connell, Phys. Rev. 180, 1289 (1969).

[19] J. Matese and R. F. O'Connell, Nature (London) 222, 649 (1969); Astrophys. J. 160, 451 (1970).

[20] D. Grasso and H. R. Rubinstein, Astropart. Phys. 3, 95 (1995); Phys. Lett. B 379, 73 (1996).

[21] J. D. Barrow, P. G. Ferreira, and J. Silk, Phys. Rev. Lett. 78, 3610 (1997).

[22] Which need not be guaranteed in the presence of noncommutativity. More precisely, NCU(1) theory, unlike its commutative counterpart, is an interacting theory, i.e., in the action and therefore in the Hamiltonian besides the terms quadratic in fields we have some terms of power three and four. This will change the $T^{2}$ behavior at temperatures higher than the noncommutativity scale.

[23] S. Weinberg, Phys. Rev. D 9, 3357 (1974).

[24] C-S. Chu, B. R. Greene, and G. Shiu, Report No. hepth/0011241.

[25] D. J. Chung, E. W. Kolb, and A. Riotto, Phys. Rev. D 60, 063504 (1999)

[26] M. M. Sheikh-Jabbari (to be published). 